\title{
Transient and local illumination of an organic photoconductive sensor
}

\author{
${ }^{1,2}$ Wouter Woestenborghs, ${ }^{1,2}$ Patrick De Visschere, ${ }^{1,2}$ Filip Beunis, \\ ${ }^{3}$ Arnout Vetsuypens, ${ }^{1,2}$ Kristiaan Neyts \\ ${ }^{1}$ Liquid Crystals \& Photonics Group, Ghent University, \\ Sint-Pietersnieuwstraat 41, 9000 Ghent, Belgium \\ ${ }^{2}$ Center for Nano and Biophotonics, Ghent University \\ ${ }^{3}$ Barco NV, Healthcare Division, President Kennedypark 35, \\ 8500 Kortrijk, Belgium
}

\begin{abstract}
In this paper we investigate the performance of a transparent photoconductive sensor based on a double layer of organic materials (m-MTDAB / PTCBI) which are deposited on two interdigitated transparent ITO electrodes. The performance of the sensor is demonstrated with electro-optical measurements: the $\mathrm{I}(\mathrm{V})$ curves consist of two linear sections meeting at a knee voltage $V_{t}$. Linear regression performed on the $I(V)$ curves below $V_{t}$ show that the conductance is a power law of the luminance incident on the device. We present a model to describe the behaviour of the sensor below $\mathrm{V}_{\mathrm{t}}$.

We present measurements of $\mathrm{I}(\mathrm{t})$ for a transient illumination of the sensor. Plotting the inverse of the current as a function of time we find that the transient is consistent with the model for voltages below $V_{t}$. For voltages above $V_{t}$ we find that the sensor behaves like a resistor in series with a space charge (SC) region. We present a local illumination experiment that confirms the existence of a $\mathrm{SC}$ region between the electrodes of the photoconductive sensor for $\mathrm{V}>\mathrm{V}_{\mathrm{t}}$. The space charge region is located near the cathode of the sensor.
\end{abstract}

Keywords : Organic electronics, photoconductive sensor, transparent, carrier lifetime

\section{INTRODUCTION}

The technological advantages of organic electronics, compared to inorganic semiconductor devices are well known. In production the organic devices promise to be low cost because manufacturing can be performed using wet techniques ${ }^{[} 1^{\text {] }}$ and/or roll-to-roll processing ${ }^{[}{ }^{3}$. From the application point of view, the properties of organic materials allow to develop novel devices with interesting features. Some examples of organic devices with special features are transparent/flexible OLED's ${ }^{[}{ }^{]}$and thin film transistors ${ }^{[}{ }^{]}$. We have published the properties of an organic photoconductive sensor ${ }^{[} 5^{]}$which is highly transparent for the same wavelengths it detects. The sensor is based on the chemical vapour-phase detector presented by John C. Ho ${ }^{[} 6^{]}$but using different organic and electrode materials to achieve optimal transparency .

In this contribution we start by summarizing the architecture of the transparent sensor. We further elaborate on the working principle of the sensor by interpreting the electro-optical measurements. The transient behaviour of the sensor is presented by measuring the $\mathrm{I}(\mathrm{t})$ curves while applying a light pulse, for a constant voltage. The transient measurements shed light on the transport of charge carriers in the sensor. A simplified model for the sensor is proposed that explains roughly the behaviour of the current as a function of the voltage. Based on the new insights we re-evaluate the local illumination experiment which was presented in our previous publication $5^{[}$. 


\section{EXPERIMENTAL PROCEDURES}

The photoconductive sensor has a lateral architecture. As a substrate we use an indium tin oxide (ITO) coated float glass substrate of $1.1 \mathrm{~mm}\left(\mathrm{R}_{\mathrm{ITO}}=100 \Omega / \mathrm{sq}\right.$, Delta Technologies). The two electrodes are patterned in the ITO layer by photolithography, the electrode fingers are oriented parallel to each other separated by a constant gap. Typically the electrodes are interdigitated to obtain a large effective length on a limited surface area. In this work the ITO electrodes are $80 \mu \mathrm{m}$ wide and separated from each other by a gap of $20 \mu \mathrm{m}$. For the local illumination experiment the length of the sensor is $10 \mathrm{~mm}$, in case of interdigitated electrodes the effective length of the sensor is several meters. The ITO electrodes are covered with a stack of two organic materials: a hole transport layer (HTL) and an exciton generation layer (EGL). The organic materials are deposited by IPMS Fraunhofer (Dresden, Germany). The device presented in this paper has an organic stack consisting of 40nm 1,3,5-Tris[(3-methylphenyl)phenylamino]benzene (m-MTDAB, Sigma Aldrich) and 10nm 3,4,9,10-perylenetetracarboxylic bis-benzimidazole (PTCBI, Sensient), respectively HTL and EGL. The hole mobility in m-MTDAB is approximately $\mu_{\mathrm{h}}=3 \times 10^{-3} \mathrm{~cm}^{2} / \mathrm{Vs}^{\left[7^{7}\right.}$. The electron mobility $\mu_{\mathrm{n}}$ of PTCBI is, according to Dhagat et. al. ${ }^{[8]}$ a function of the orientation of the PTCBI molecules in the EGL, and ranges from $2 \times 10^{-2} \mathrm{~cm}^{2} / \mathrm{Vs}$ to $2 \times 10^{-4} \mathrm{~cm}^{2} / \mathrm{Vs}$.

The photoconductive sensor is encapsulated with a cover glass. The cover glass is etched at the level of the sensor to create a cavity, next to the sensors $\mathrm{CaO}$ getters are positioned which fit in the encapsulation cavity. In a nitrogen environment, the cover glass is glued to the sensor substrate with UV curable glue. The encapsulation is performed by IPMS Fraunhofer directly after evaporation of the organic stack. Figure 1 shows a cross section of the photoconductive sensor, perpendicular to the ITO electrodes.

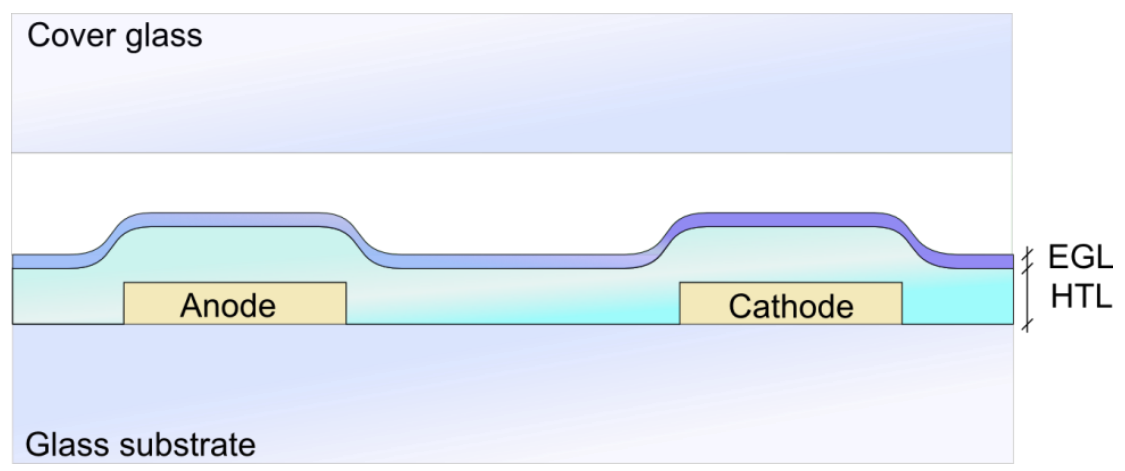

Figure 1 Cross section of the organic photoconductive sensor encapsulated with an etched cover glass. The cavity is filled with $\mathrm{N}_{2}$.

To determine the spectral absorption of m-MTDAB and PTCBI we deposit a single layer of each on bare glass substrates. To measure the thickness of the deposited layers we use a profilometer (WYKO NT3300). The transmission of the single layer samples is measured with a photospectrometer (Perkin-Elmer Lambda 35), the reference sample is an uncoated bare substrate.

The electro-optical measurements demonstrate the functionality of the sensor by measuring the current as a function of the applied voltage and as a function of the irradiation of the sensor. To illuminate the sensor it is placed on a LED backlight which is driven with a current source (Keithley 220). The luminance of the LED backlight is measured with a Chroma 5 Colorimeter (X-Rite). The sensor is placed in series with a current meter (Keithley 6485 PicoAmpMeter) and the circuit is driven with a voltage source (Keithley 2425 SourceMeter). A LabView program controls the measurement equipment and performs the $\mathrm{I}(\mathrm{V})$ measurement for different luminance levels of the LED backlight.

To measure the current I through the sensor as a function of time $t$, we use a current-voltage amplifier (Stanford Research Systems SR570) in combination with a digital oscilloscope (Tektronix TDS540A). The voltage signal across the 
electrodes is provided by an arbitrary wave generator (Tektronix AFG5101). The illumination source and the luminance detection is similar as in the I(V) setup. To synchronize the voltage, the oscilloscope and the LED backlight control, a data acquisition card (National Instruments USB 6212) is used. In this work we study the response of the sensor to a pulse of light, keeping the electrodes at a constant voltage.

For the local illumination experiment the photoconductive sensor is placed on a microscope stage (Nikon Ti Eclipse) on an optical table. On the optical table a laser beam is shaped into a line and coupled into the microscope so that a narrow laser line is projected on the photoconductive sensor parallel to the electrodes, and in addition the sensor is illuminated by the microscope light. An extensive discussion of the microscope setup is published in previous work ${ }^{[} 5^{]}$. The sensor is connected to a current meter and voltage source as in the $\mathrm{I}(\mathrm{V})$ measurements. The local illumination experiment allows to measure the current through the photoconductive sensor I as a function of the position $\mathrm{x}$ of the local illumination, while the applied voltage is fixed. Note that for each $\mathrm{x}$ position the current through the sensor is measured with and without the laser line on the sample. The projected laser line is approximately $1 \mathrm{~mm}$ long and has a Gaussian distribution with a variance $\sigma$ of approximately $1 \mu \mathrm{m}$.

\section{RESULTS \& DISCUSSION}

The spectral transmission measurements show that m-MTDAB is transparent in the visible spectrum with more absorption towards the UV. The PTCBI shows significant absorption in the green and red (Figure 2). The thickness of the single organic layers is measured with the profilometer at the edge of the substrate.

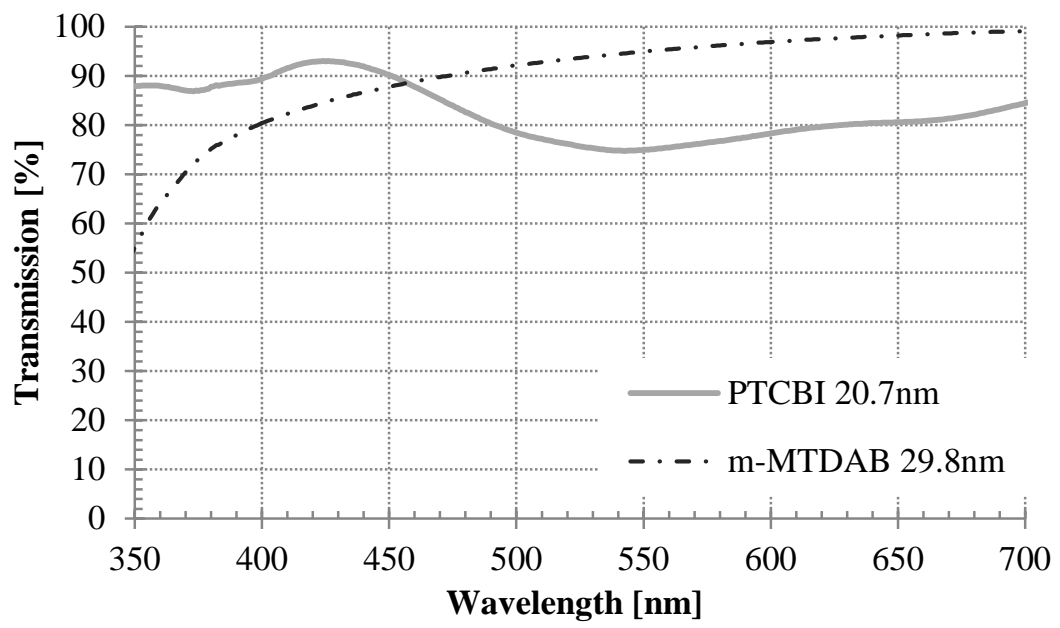

Figure 2 The spectral transmission measurements for a single layer of m-MTDAB $(29.8 \mathrm{~nm})$ and a single layer of PTCBI (20.7nm), deposited on a bare glass substrate.

The spectral absorption coefficient of PTCBI is calculated from the transmission spectrum taking into account the actual thickness of the layer and neglecting the reflection at the interface glass/PTCBI and PTCBI/air. For the wavelength of the laser $(635 \mathrm{~nm})$ in the local illumination setup, we find an absorption coefficient of $4.610^{6} \mathrm{~m}^{-1}$.

The efficiency with which an exciton is generated by absorption of photons is $\gamma_{\mathrm{Gen}}$. Typically excitons are strongly bound ${ }^{[}{ }^{]}$, and the probability for them to dissociate into free charge carriers is small. Via molecular interaction excitons can however diffuse through the organic layer towards the EGL/HTL interface where dissociation into charge carriers is simplified. The diffusion efficiency $\gamma_{\text {Diff }}$ is the probability that a generated exciton reaches the EGL/HTL interface. At the EGL/HTL interface an exciton moves over to the charge transfer state ${ }^{[} 10^{1}$ (CT state), in that state the electron remains in the EGL but the hole is transferred to the HTL. In the CT state, the electron and the hole are bound across the interface via Coulomb forces. The probability with which the CT state dissociates into free charge carriers is close to unity ${ }^{[} 11^{]}$. 
The electro-optic performance of a sensor sample (length $=2.384 \mathrm{~m}$ ), for 4 luminance levels $\mathrm{L}_{\mathrm{v}}$ and in the dark, is shown by means of $\mathrm{I}(\mathrm{V})$ curves in Figure 3.

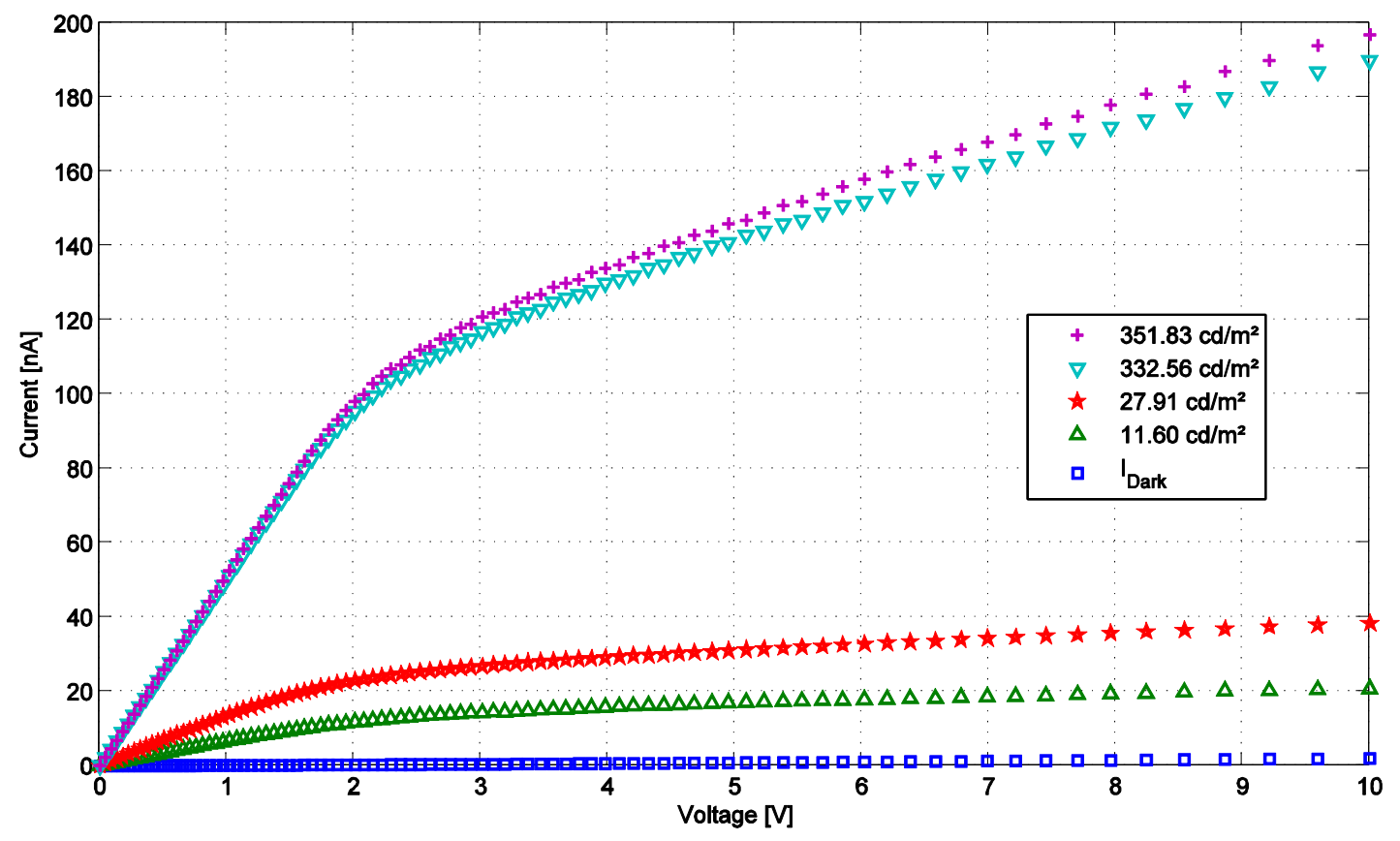

Figure 3 Current measurement as a function of voltage for four luminance levels and the dark current .

The plot shows that the contrast between the curves is a function of the illumination, but the relation is not linear. In general the $\mathrm{I}(\mathrm{V})$ curves show two linear regimes separated by a transition voltage $\mathrm{V}_{\mathrm{t}}$. Below $\mathrm{V}_{\mathrm{t}}$ the sensor acts as a resistor with a resistivity that is a function of the illumination of the sensor. The conductance $G$ of the sensor depends linearly on the charge carrier densities $n$ and $p\left[1 / \mathrm{m}^{2}\right]$ in the organic layers. Assuming there is no net charge and the sensor is in equilibrium, then the electron and the hole density are equal and the continuity equation is written as:

$$
\frac{\partial n}{\partial t}=g-\beta n^{\alpha}
$$

Here $\mathrm{n}$ is the areal electron density $\left[1 / \mathrm{m}^{2}\right], \mathrm{g}$ the rate at with which charge carriers are generated $\left[1 / \mathrm{sm}^{2}\right], \beta$ the recombination constant $\left[\mathrm{m}^{2(\alpha-1)} / \mathrm{s}\right]$ and $\alpha$ the recombination exponent. Note that in general the recombination is a function of both areal carrier densities $n$ and $p$, but in equilibrium $n=p$. We assume that the generation rate $g$ is independent of the charge carrier densities and linearly related to the luminance of the LED backlight $\left(g=\gamma_{\text {Diff }} \gamma_{G e n} L_{v}\right)$. In steady state the solution of the differential equation is then:

$$
G \sim n=\left(\frac{g}{\beta}\right)^{1 / \alpha}
$$


Equation (2) shows we then expect the conductance $G$ to be proportional with the generation $g$ to the power $(1 / \alpha)$. Applying linear regression to the $\mathrm{I}(\mathrm{V})$ curves below $\mathrm{V}_{\mathrm{t}}$, we can plot the conductance of the sensor as a function of the luminance (Figure 4).

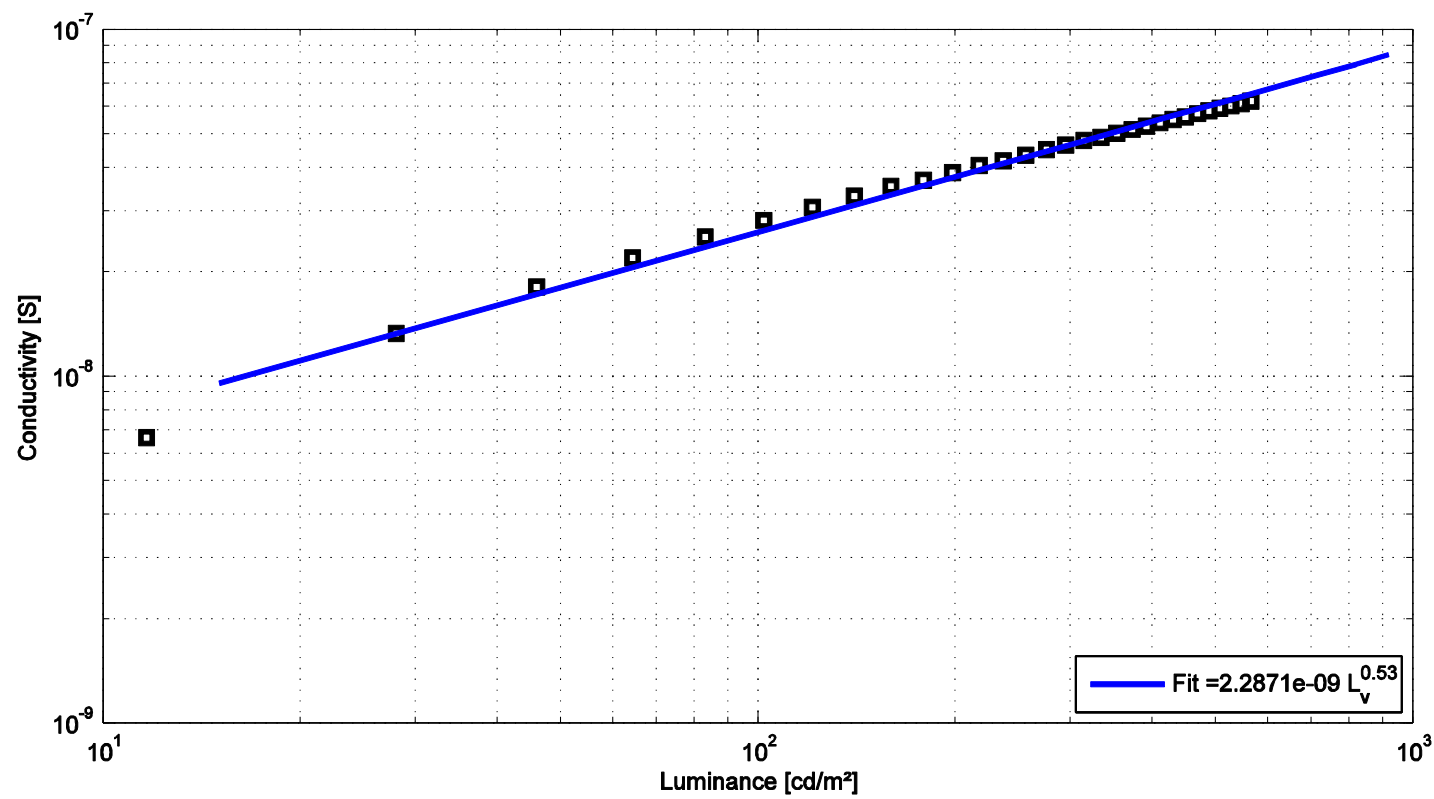

Figure 4 Conductance of the photoconductive sensor for a voltage below $V_{t}$, as a function of the luminance $L_{v}$ of the LED backlight.

On the double logarithmic plot the $G\left(L_{v}\right)$ curve forms a more or less straight line. Fitting a power law to the data points we obtain an exponent of 0.54 for this sample, compatible with the choice $\alpha=2$. Note however that a similar analysis of the samples of our previous publication ${ }^{[}{ }^{]}$yields fitting exponents between 0.19 and 0.36 for the conductivity below $V_{t}$, hence the $\alpha$ for those cases is higher. The deviation of $\alpha$ may be due to additional recombination of charge carriers at traps in the organic materials. At higher $\mathrm{L}_{\mathrm{v}}$ the plot shows that the slope of the data points is reduced compared to the fitting curve, this may be due to exciton quenching in the EGL, similar to the mechanism proposed by Yichun Lou et. al. ${ }^{[} 12^{]}$. Another possibility is that the efficiency of charge separation at the interface depends on the concentration of the excitons.

We have studied the current $\mathrm{I}(\mathrm{t})$ for a transient in the illumination. When the sensor is in steady state and the applied luminance $\mathrm{L}_{\mathrm{v}}$ is suddenly removed, the generation term $\mathrm{g}$ in equation (1) becomes zero. As solution for the differential equation we find :

$$
\frac{1}{n^{\alpha-1}}-\frac{1}{n_{0}{ }^{\alpha-1}}=(\alpha-1) \beta t
$$

For the simple case where $\alpha=2$ and dividing by $q\left(\mu_{n}+\mu_{p}\right) E$, we find a relation between the current density $\mathrm{J}$ and the time t:

$$
\frac{1}{J}=\frac{1}{J_{0}}+\frac{\beta t}{q\left(\mu_{n}+\mu_{p}\right) E}
$$

We measure the $I(t)$ of the sensor sample for five voltages below and six voltages above $V_{t}$. For each voltage we repeat the measurements for six luminance levels. Typical $\mathrm{I}(\mathrm{t})$ measurements are presented in Figure 5 where the inverse of the current is plotted as a function of the time $\mathrm{t}$ for an applied voltage of $0.4 \mathrm{~V}$ and $6 \mathrm{~V}$, both for $\mathrm{L}_{\mathrm{v}}$ approximately $80 \mathrm{~cd} / \mathrm{m}^{2}$ and $470 \mathrm{~cd} / \mathrm{m}^{2}$. 


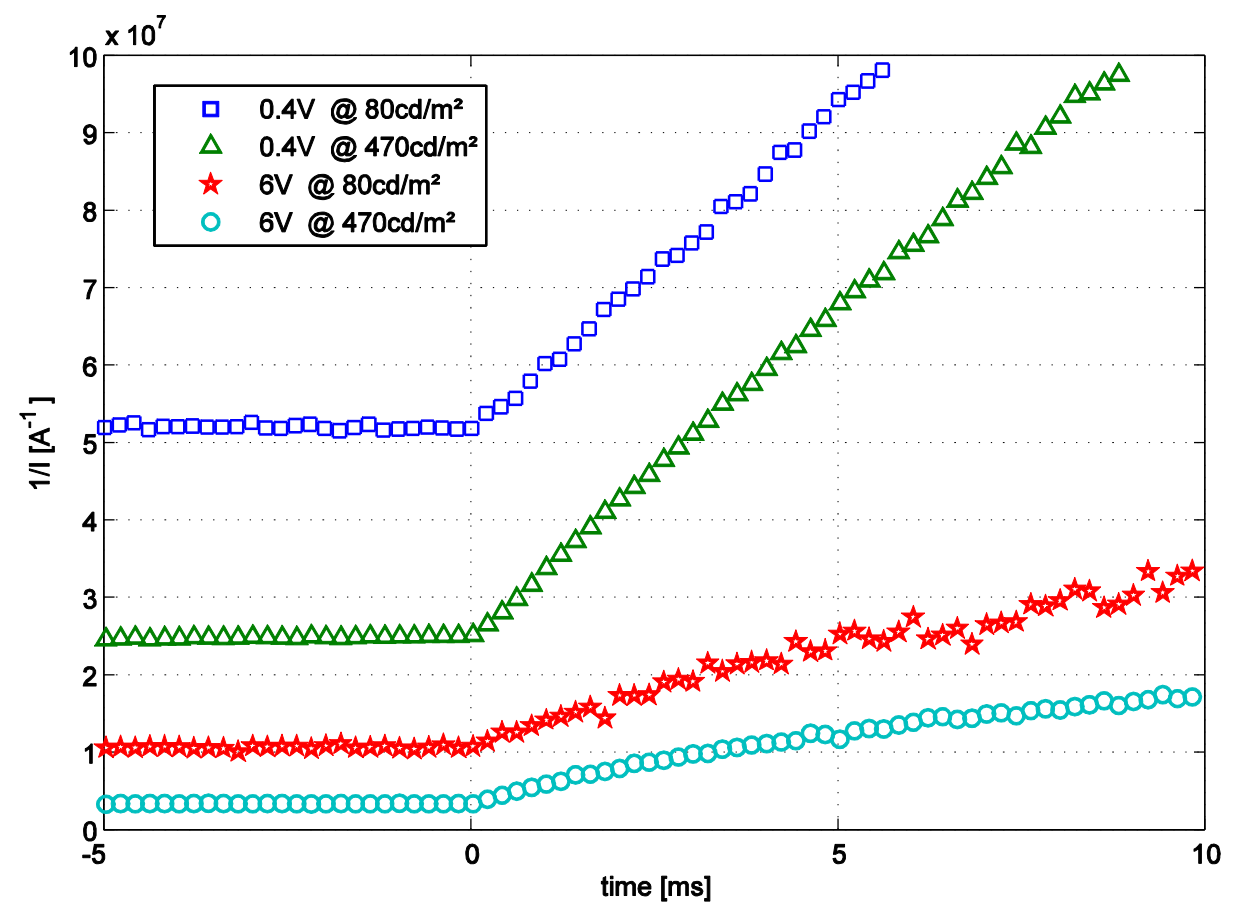

Figure 5 The inverse of the current as a function of time for the voltages $0.4 \mathrm{~V}$ and $6 \mathrm{~V}$, at $\mathrm{L}_{\mathrm{v}}$ of approximately $80 \mathrm{~cd} / \mathrm{m}^{2}$ and $470 \mathrm{~cd} / \mathrm{m}^{2}$.

The plots show that the transient $(t>0)$ of the inverse current is a linear function of the time. When we compare the plots for the same voltage, we notice that the slope of the transient is independent of the luminance level. The slope is however a function of the applied voltage. We calculate the transient slopes by performing linear regression between $0.2 \mathrm{~ms}$ and $1 \mathrm{~ms}$. Figure 6 presents the slopes as a function of the applied voltage on a double logarithmic plot.

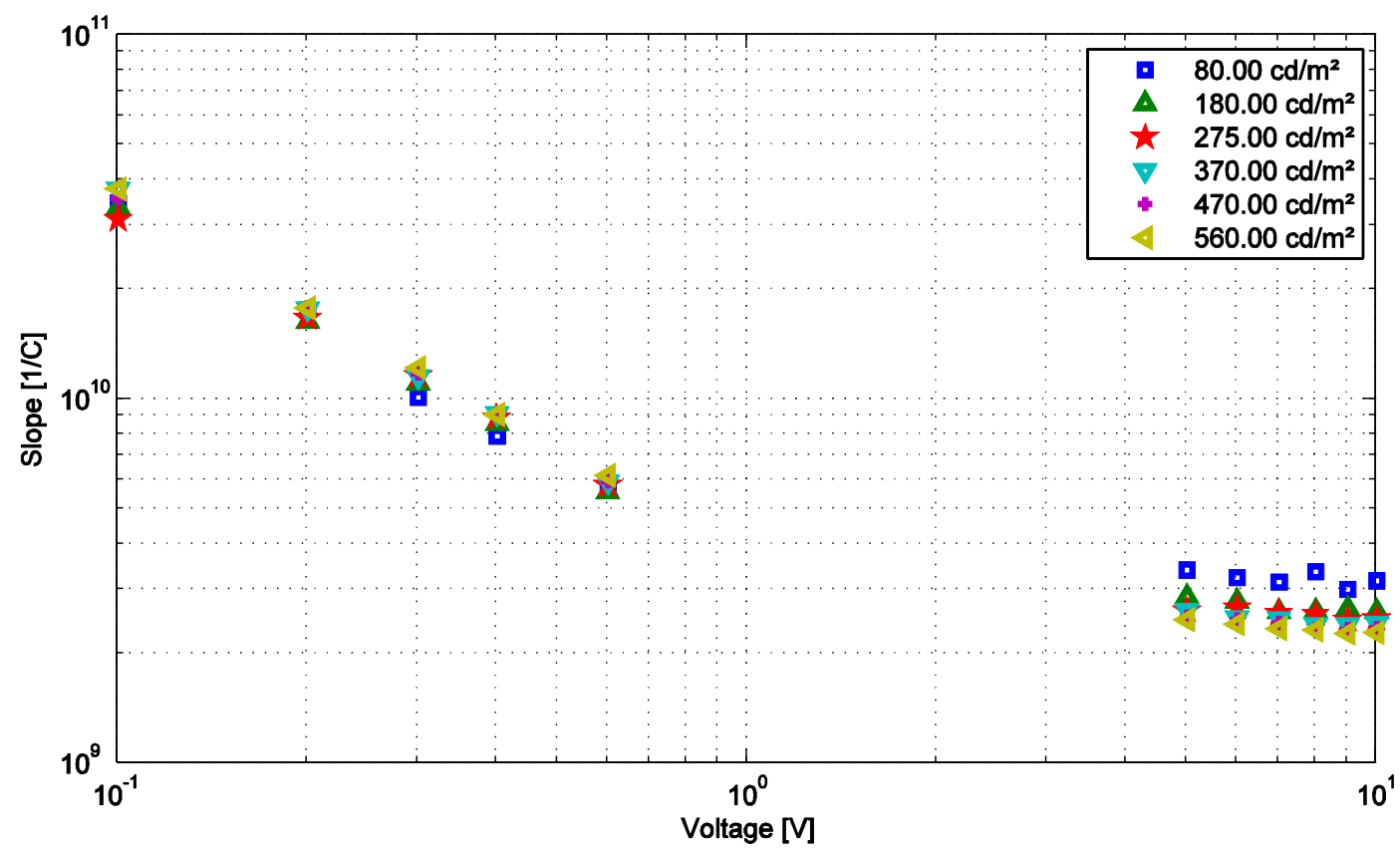

Figure 6 Slope of the inverse current curves for $t>0$ as a function of the applied voltage. 
The plot shows that below $V_{t}$ the slopes are inversely proportional to the applied voltage. When we fit a power law function to the data points below $\mathrm{V}_{\mathrm{t}}$ (for each of the luminance levels) the exponent of the fit ranges from -0.94 to -1.09 . This shows that below $V_{t}$ the transient measurements respond to a luminance step as described in equation (3). Below $V_{t}$ the sensor behaves as a conventional photoconductive sensor. Above $\mathrm{V}_{\mathrm{t}}$ the transient slope does not longer decrease with the applied voltage, but stays approximately equal to the slope at $\mathrm{V}_{\mathrm{t}}$. This can be explained by the occurrence of a space charge (SC) region in part of the gap. The remaining part of the gap will still behave as a resistor and since (4) can still be applied to that part, the voltage over this resistive region must approximately be equal to Vt. The remaining $\mathrm{V}$ - Vt is then taken up by the space charge region. The plot thus suggests that the sensor consists of a photoconductive sensor in series with a space charge region (SC).

In our previous work[5] we presented the current $\mathrm{I}(\mathrm{x})$ through the sensor as a function of the position $\mathrm{x}$ of a local illumination with a laser beam with a line-like profile parallel to the electrodes. The $I(x)$ curve for a voltage above $V_{t}$ is presented in Figure 7.

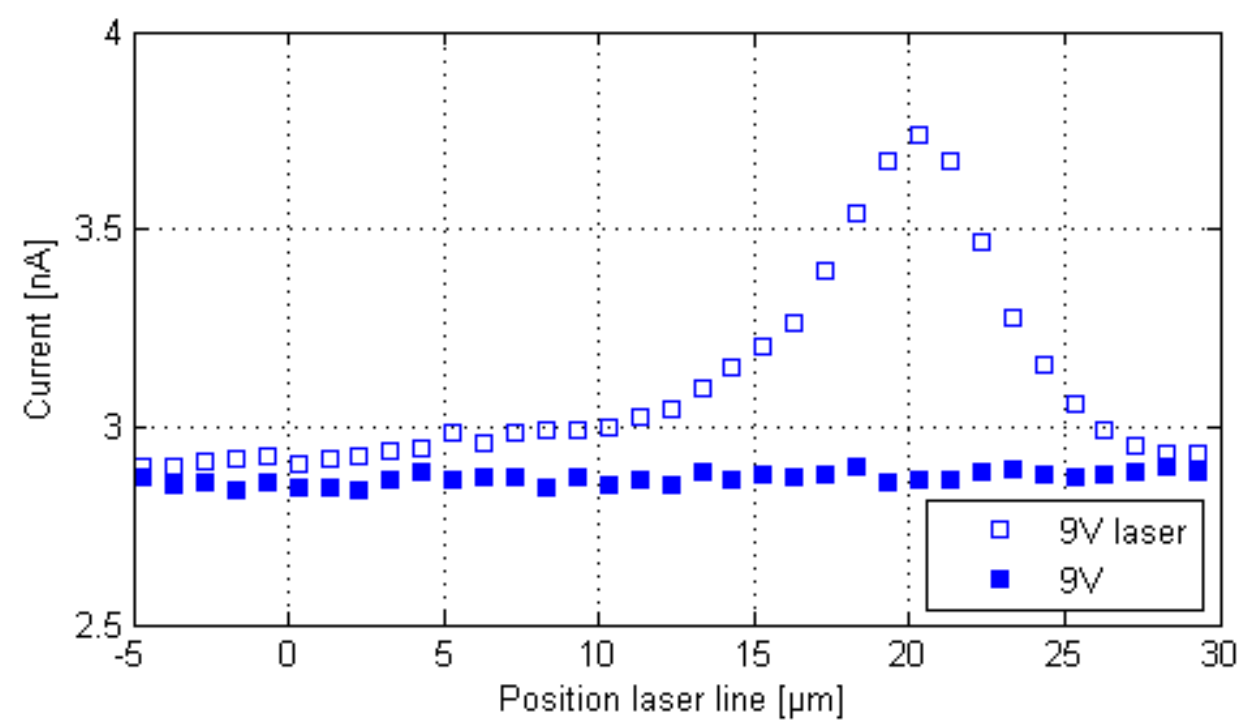

Figure 7 Current $I$ through the sensor as a function of the position $x$ of the local illumination.

The $\mathrm{I}(\mathrm{x})$ curve shows that the sensitivity of the sensor for an applied voltage above $\mathrm{V}_{\mathrm{t}}$ is a function of the position of the laser beam. This is compatible with the proposed occurrence of a SC region to explain the transient current measurements. In the SC region carrier densities are low and recombination is also low, leading to a high collection efficiency for generated carriers. Since the increase of the sensitivity is located near the cathode the SC is positive.

\section{CONCLUSION}

In this contribution we analyzed the functionality of a double layered organic photoconductive sensor. With the electrooptical measurements we demonstrated the performance of the sensor and showed that the conductance is a non linear function of the illumination. The $\mathrm{I}(\mathrm{V})$ curves suggest that below the threshold voltage $\mathrm{V}_{\mathrm{t}}$, the sensor behaves like a light dependent resistor.

To explain the resistivity of the sensor as a function of $\mathrm{L}_{\mathrm{v}}$, for voltage $\mathrm{V}<\mathrm{V}_{\mathrm{t}}$, we proposed a model based on the law of charge conservation in equilibrium. We studied the transient behaviour of the sensor for a light pulse by measuring the $\mathrm{I}(\mathrm{t})$ curve. The measurement was presented as the inverse of the current as a function of time to compare with the proposed model. We found that the transient measurements are consistent with the proposed model for $\mathrm{V}<\mathrm{V}_{\mathrm{t}}$.

Above $\mathrm{V}_{\mathrm{t}}$ we showed that the simple model is no longer sufficient to describe the behaviour of the photoconductive 
sensor. The time dependence of the sensor current for $\mathrm{V}>\mathrm{V}_{\mathrm{t}}$ shows a rapid decay which is no longer a function of the applied voltage. Supported by the local illumination experiment of our pervious publication[5] we model the sensor at voltages above $\mathrm{V}_{\mathrm{t}}$ as a resistor in series with a high field space charge region.

\section{ACKNOWLEDGMENT}

This work is a result of the TARDIS research \& development project funded by the IWT (Institute for the Promotion of Innovation by Science and Technology in Flanders), it is also the result of collaboration within the IAP (Interuniversity Attraction Poles Programme - Belgian State - Belgian Science Policy).

\section{REFERENCES}

[1] F. F. Yu, H. L. Fan, H. F. Huang, Q. Y. Cao, Y. F. Dai, X. C. Gao, Y. Z. Shang, M. Y. Zhang, L. Long, H. Xu, X.F. Li, and B. Wei, "Blue fluorescence from the ligand and yellow phosphorescence from the iridium complex: Highefficiency wet-processed white organic light-emitting device," Inorganica Chimica Acta 390, pp. 119-122, July 2012.

[2] I. Bose, A. Ohlander, M. I. J. Stich, C. Kiesl, D. Hemmetzberger, G. Klink, S. Trupp, and K. Bock, "Polymer optochemical-electronic based module as a detection system for volatile analytes on a foil substrate," 2012.

[3] S. M. Lee, C. S. Choi, K. C. Choi, and H. C. Lee, "Low resistive transparent and flexible zno/ag/zno/ag/wo3 electrode for organic light-emitting diodes,” Organic Electronics 13, pp. 1654-1659, Sept. 2012.

[4] H. Ohta, T. Kambayashi, K. Nomura, M. Hirano, K. Ishikawa, H. Takezoe, and H. Hosono, "Transparent organic thin-film transistor with a laterally grown non-planar phthalocyanine channel," Advanced Materials 16, pp. 312-+, Feb. 2004.

[5] W. Woestenborghs, P. De Visschere, F. Beunis, G. V. Steenberge, K. Neyts, and A. Vetsuypens, "Analysis of a transparent organic photoconductive sensor," Organic Electronics 13(11), pp. 2250 - 2256, 2012.

[6] J. C. Ho, A. Arango, and V. Bulovic, "Lateral organic bilayer heterojunction photoconductors," Applied Physics Letters 93, p. 063305, Aug. 2008.

[7] Y. Shirota, "Organic materials for electronic and optoelectronic devices," Journal of Materials Chemistry 10(1), pp. 1-25, 2000.

[8] P. Dhagat, H. M. Haverinen, R. J. Kline, Y. Jung, D. A. Fischer, D. M. DeLongchamp, and G. E. Jabbour, "Influence of dielectric surface chemistry on the microstructure and carrier mobility of an n-type organic semiconductor," Advanced Functional Materials 19, pp. 2365-2372, AUG 102009.

[9] M. Knupfer, "Exciton binding energies in organic semiconductors," Applied Physics A-materials Science \& Processing 77, pp. 623-626, Oct. 2003.

[10] K. Gao, S. J. Xie, S. Yin, and D. S. Liu, "Study on charge-transfer state in a donor-acceptor polymer heterojunction," Organic Electronics 12, pp. 1010-1016, June 2011.

[11] S. I. Choi, J. Jortner, S. A. Rice, and R. Silbey, "Charge-transfer exciton states in aromatic molecular crystals," Journal of Chemical Physics 41(11), pp. 3294-\&, 1964.

[12] Y. C. Luo, H. Aziz, G. Xu, and Z. D. Popovic, "Electron-induced quenching of excitons in luminescent materials," Chemistry of Materials 19, pp. 2288-2291, May 2007.

Wouter.Woestenborghs@elis.ugent.be

http://lcp.elis.ugent.be 